\title{
A Mini Micro-electrical Stimulation Device Designed to Heal Dental Implant
}

\section{Yuxuan Gao}

The Chinese People's Liberation Army General Hospital, Beijing, China

Liyong Yang

The Northern Industrial University, Beijing, China

Xiaoyu Wang, Hongbo Li*

The Chinese People's Liberation Army General Hospital, Beijing, China

\begin{abstract}
Aim: This paper introduces a design method of implant - bone healing device. Methods The cathodic of the device contacts to the surface of the dental implant with metal retaining bolts on it, and provides electrical stimulation through the cathode and anode, respectively, to a dental implant and other parts of the oral cavity. The device is in a small size. In a dental implant operation, it can be disassembled conveniently, and is easy to use and clean, and also safe and reliable. Result: We have completed the equipment production and run successfully, conditions and basis for clinical trials.
\end{abstract}

KEYWORD: Micro dental device; Physical therapy

\section{INSTRUCTIONS}

In recent years, dental implant prosthesis is a hotspot in clinical and medical researchers. To improve the healing level of implant - bone tissue is the main challenge for scholars. The migration of cells to the implant - bone interface, proliferation and deposition of bone matrix is the key to successful implant bones. Since the beginning of 1980s, there had been a lot of research on the biological effects of the electric field, and the field has also been widely used in the biomedical field, including promotion of fracture healing, electrotherapy tumors, trauma, muscle contraction function and nerve growth areas (Nina Tandon et al.2011, Yun Xiang et al.2014 Paweł Hottowy et al.2012). However, there are only few reports on the application of electrical stimulation to promote oral dental implant healing.

The micro-device we designed to stimulate the healing of dental implant is using a cathode to contact the surface of the dental implant, while the cathode is fixed on the dental implant by retaining metal bolt that contacted with it, and an anode disposed in contact with other parts of the oral cavity such as soft tissue. The device provides electrical simulation through the cathode and anode, respectively, to a dental implant and other parts of the oral cavity. The retaining screw is sealed by the dental resin, and protecting tissues through insulating layer. The device is non-invasive, and easy to control the time and degree of treatment. It is also easy to disassemble and use without causing inconvenience to the patient's oral environment. Overall it has very important application value.

\section{MECHANISM}

After tissue injured, the device can form a stable endogenous electric fields (about 100-200mv) between the damaged tissue and non-damaged tissue (Angel V Peterchev et al.2012). The electric field will last for a long duration (several days to several weeks), and distributes around the damaged tissue within several millimeters, the closer the distance to tissue damage is, the stronger the electric field is (Yoshihiro Maruyama et al.2012). It always generated new bone deposition at a constant negative potential, and tend to produce bone resorption at the positive potential, which means displaceable charges may be reveal the significance of bone growth (Jayme S. Knutson et al.2013). Appling external electric field to dental implants can improve the migration of stem cells (Mohamadreza Baghaban Eslaminejad et al.2013), which may promote healing process around the implant and improve the success rate. According to reports, the low-frequency microelectric field can also inhibit the growth of bacteria, which can reduce the probability of infection of wound healing.

\section{DESIGN AND IMPLEMENTATION}

\subsection{The healing apparatus comprising}

The healing apparatus comprising power supply, micro-voltage constant-voltage circuit module. Controlling the electrodes provide voltage below $5 \mathrm{~V}$. Micro-current constant-current circuit module. 
Controlling the electrodes provide current between $10-200 \mu \mathrm{A}$.

Electrode, comprising a cathode (6) and an anode (5), is used to export and provide the current and voltage respectively to implants and other parts of the body in the mouth from the micro-current constant-current circuit or micro-voltage constantvoltage circuit. An insulating protective layer 3 is provided on the outer surface of the healing device, so that the electronic components are segregate from the oral environment.

Micro-voltage constant-voltage circuit module and micro-current constant-current circuit module do not co-exist, one of the two modes must be chosen during the process.

The cathode (6) set in the lower surface of the contact between implant and the device, and the outer surface of screw of retaining bolt (1) which above the lower surface of the healing device. The anode (5) set in the contact projecting portion. The cathode (6) and the anode (5) are connected to the insulating protective layer (3) respectively.

Mini micro-electrical stimulation device comprises a round barrel body and an out of contact part (Figure 1, 2 and 3). The body portion is used to install, fix to the dental implant, and accommodate the circuit module. Out parts of the device is used to contact with other parts of the mouth, such as soft tissue.

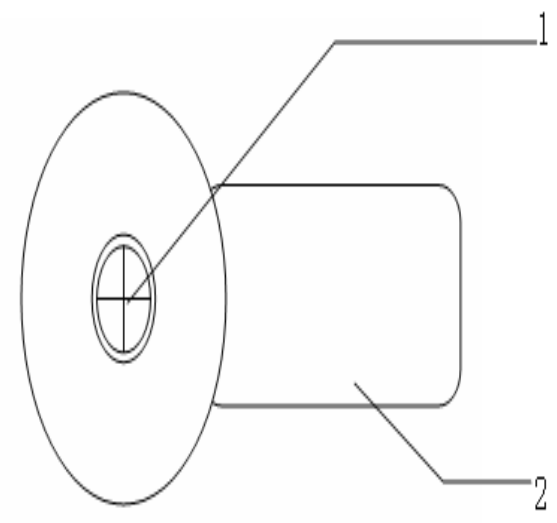

Figure 1. A schematic top view of mini micro-electrical stimulation device. 1-Retaining bolt,2-Contact portion extending.

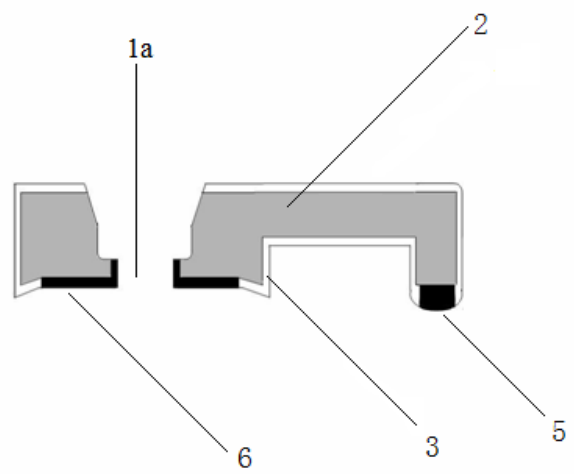

Figure 2. A schematic profile structure of mini micro-electrical stimulation device.1a-Through hole, 2-Contact portion extending, 3-The insulation layer, 5-Anode, 6-Cathode.

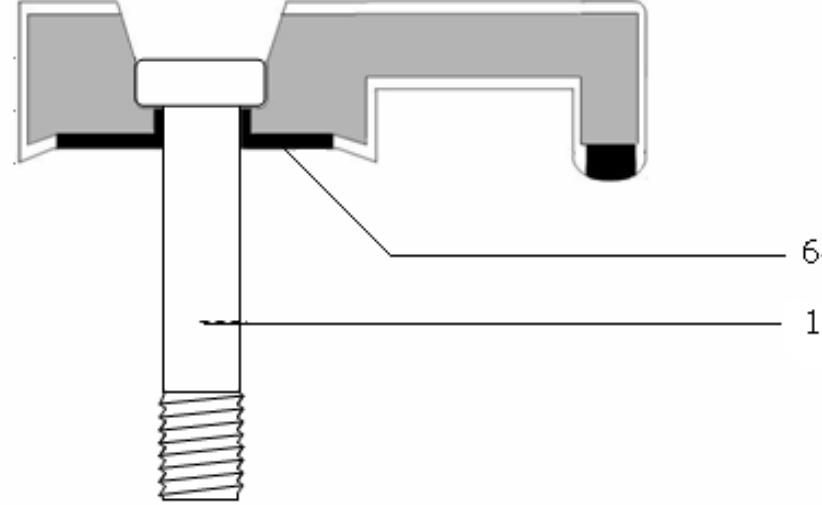

Figure 3. A schematic profile structure of mini micro-electrical stimulation device. 1-Retaining bolts,6-Cathode.

The device is fixed to the implant surface by retaining bolt (1), so that the device can be stably secured to the implant surface in the healing process, and avoiding the device displacement during the treatment. The device is preferred titanium or titanium alloy retaining bolt to improve the biocompatibility.

In addition to retaining screw fixation means serve to heal, it also acts as an electrically conductive, since the bolts retaining the implant in contact with the cathode, the cathode output electrical signal can be further introduced into the implant, especially when the cathode poor contact with the implant (Figure 4).

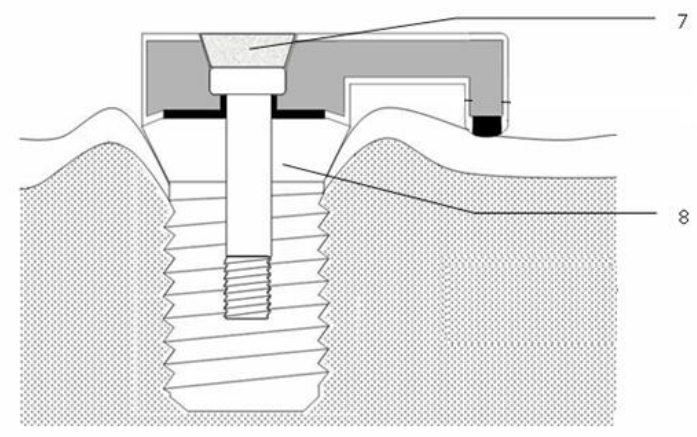

Figure 4. The installation structure diagram of mini microelectrical stimulation device. 7-Dental resin,8-Dental Implants.

The surface of dental resin(7) has the same altitud e as that of other parts of the device, so as to prevent damage and insulate to the patient's oral cavity.

The insulation protection layer(3) is set on the sur face of the healing device and the extending contact portion consists of lateral contact parts and longitudinal contact parts (Figure 2, 3 and 4). The lateral contact part retains a certain gap between the anode of micro-electric field analog and healing device of micro dental implant and the implant itself. The anode(5) is set at the bottom of the contact part, making it easy for anode(5) to contact with the soft ti ssue in the mouth.

As the power supply, it uses a micro cell, such as a button battery, etc. 


\subsection{Constant pressure control module}

Constant pressure control module includes (Figure 5,6 and 7):

The controller controls the operational amplifier $g$ roups by accepting signals output by operational amp lifier groups and inputting signals into operational amplifier groups so as to ensure that the operational amplifier group can output constant voltage circuit and the operation amplifier group is used to produce constant voltage circuits and send the mtothe electrode.

The direct current converter(21) converts the volt age of micro battery(4) into fixed voltage and input $i$ $\mathrm{t}$ to the operational amplifier groups, and the direct $\mathrm{c}$ urrent convert(22) converts the voltage of micro batt ery(4) into fixed voltage and input them to the contro ller.

The connection relationship of power supply mod ule (Figure5). Micro battery 4 is connected to direct current converter(21) and direct current converter (22)respectively and the output ends of direct currentconverter(21) and direct current converter(22) are the output end of the whole power supply module.

The connection relationship of power supply mod ule and constant pressure controlling module (figure 5). Direct current converter (21) is connected to operational amplifier group (24) and direct current converter(22) is connected to the controller(23).

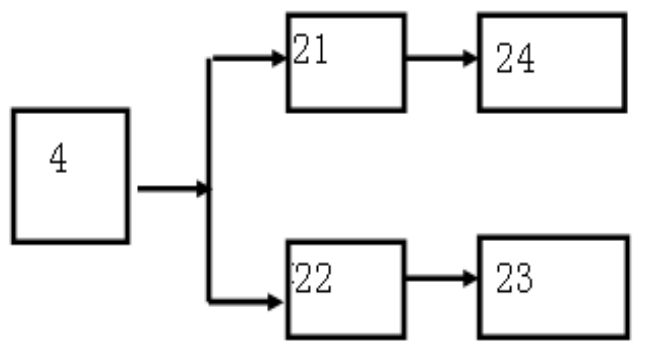

Figure 5. The structure diagram of the power supply module of mini micro-electrical stimulation device.4 -Micro Battery,21DC converter,22-DC converter,23-Controller,24-Operational amplifier group.

\subsection{The operational amplifier group}

The operational amplifier(24a)turns the signals(Vin) output by the controller into proportional voltage signal and inputs the voltage signal to the resistance(24c) as well as operational amplifier(24b). The operational amplifier(24b) receives signals from the resistance and operational amplifier(24a), output $\mathrm{s}$ signals to operational amplifier(24a) and the contro ller and realizes constant current output through clos e loop feedback. Wherein, both operational amplifier $24 \mathrm{a}$ and operational amplifier(24b) receive voltage from direct current converter (21).
In the constant pressure controlling module, the c ontroller controls the operational amplifier group by inputting signals to operational amplifier group and receiving signals from operational amplifier gro ups so as to make the operational amplifier group ou tput signals of constant voltage.

The controller includes:

The digital analog converter (23b) is used to output controlling pressure which is proportional to the set constant pressure value as the input signal (Vin) and send it to the operational amplifier (24a). The analog converter (23a) is used to receive signals from operational amplifier (24b).

The connection relationship between the controlle $\mathrm{r}$ and operational amplifier group is shown as figure 6. Digital analog converter (23b) inputs signals to the operational amplifier (24a), and another operational amplifier (24b) inputs signals to another analog converter (23a).

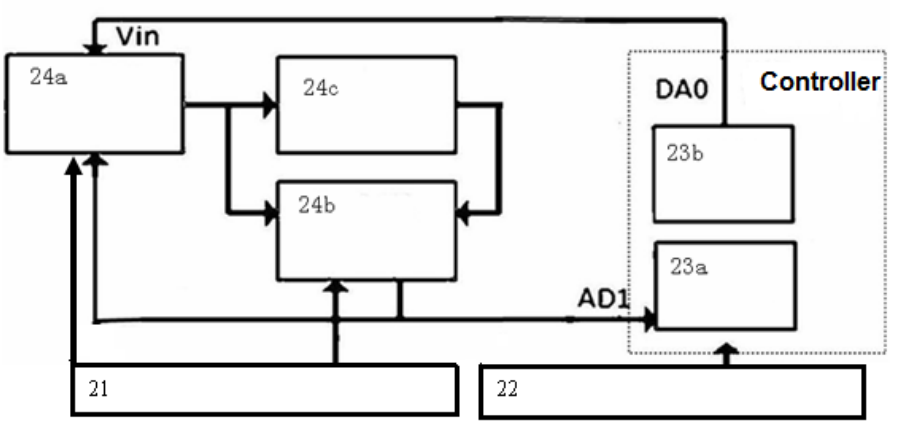

Figure 6. The structure of the constant pressure control module diagram of mini micro-electrical stimulation device. 21- DC converter, 22-DC converter analog to digital converter, 23a$\mathrm{AD}$ converter 23b-digital to analog converter, 24a-Operational Amplifier, 24b-Operational Amplifier, 24c-resistance.

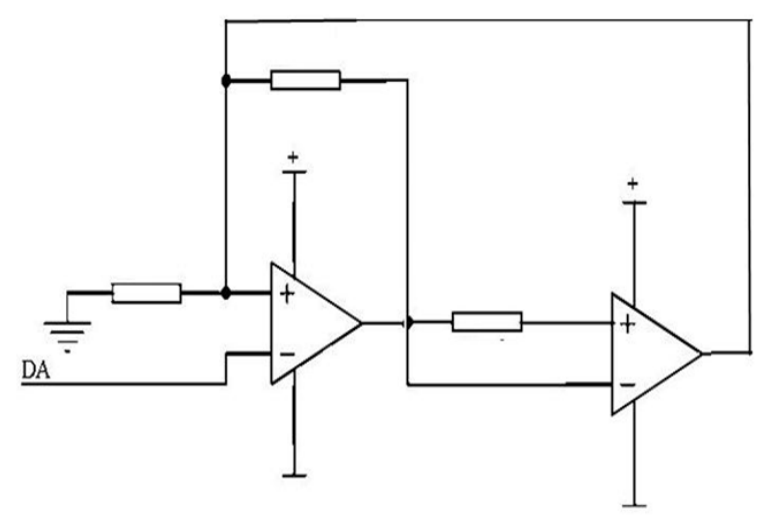

Figure 7. Micro current constant voltage control module circuit principle diagram.

\section{CONCLUSION}

After experimental verification, the proposed mini dental implant micro electrical stimulation device as a new therapy device is feasible and correct, yet its mechanism still needs further study. Expand the use of micro-electrical device to animal experiments 
proved: Microelectronics treatment can shorten the healing time of bone-implant, and provides a new method to promote healing of the implant in human beings treatment.

The miniaturization and intelligentization of medical equipment is the tendency of its development. Maximize the use of the implant attachment location can effectively reduce the system volume and power consumption; this can help saving resources and operate easily. This device is based on this principle, so that the instrument volume is smaller than the traditional ones, and has more convenience and more stable performance.

\section{ACKNOWLEDGEMENTS}

This study was supported by the Chinese Army fiveyear project funding (CWS12J134).Thanks to the Northern Industrial University provided helps in terms of equipment and developed.

\section{REFERENCES}

[1] Angel V. Peterchev, Timothy A. Wagner, Pedro C. Miranda, Michael A. Nitsche, Walter Paulus, Sarah H. Lisanby, Alvaro Pascual-Leone, Marom Bikson. 2012 Fundamentals of Transcranial Electric and Magnetic Stimulation Dose: Definition, Selection, and Reporting Practices. Brain Stimul 5(4): 435-453.
[2] Jayme S. Knutson, Kristine Hansen, Jennifer Nagy, Stephanie N. Bailey, Douglas D. Gunzler, Lynne R. Sheffler, John Chae. 2013 Contralaterally Controlled Neuromuscular Electrical Stimulation for Recovery of Ankle Dorsiflexion: A Pilot Randomized Controlled Trial in Chronic Stroke Patients. Am J Phys Med Rehabil 92(8): 656-665. Mohamadreza Baghaban Eslaminejad, Nesa Fani, Maryam Shahhoseini. 2013 Epigenetic Regulation of Osteogenic and Chondrogenic Differentiation of Mesenchymal Stem Cells in Culture. Cell J 15(1): 1-10.

[3] Nina Tandon, Anna Marsano, Robert Maidhof, Leo Wan, Hyoungshin Park, 2011 Gordana VunjakNovakovic.Optimization of Electrical Stimulation Parameters for Cardiac Tissue Engineering. J Tissue Eng Regen Med 5(6): e115-e125.

[4] Paweł Hottowy, Andrzej Skoczeń, Deborah E. Gunning, Sergei Kachiguine, Keith Mathieson, Alexander Sher, Piotr Wiącek, Alan M. Litke, Władysław Dąbrowski. 2012 Properties and application of a multichannel integrated circuit for low-artifact, patterned electrical stimulation of neural tissue. J Neural Eng 9(6):066005.

[5] Yoshihiro Maruyama, Aimi Kawano, Shizuko Okamoto, Tomoko Ando, Yoshinobu Ishitobi, Yoshihiro Tanaka, Ayako Inoue, Junko Imanaga, Masayuki Kanehisa, Haruka Higuma, Taiga Ninomiya, Jusen Tsuru, Hiroaki Hanada, Jotaro Akiyoshi. 2012 Differences in Salivary AlphaAmylase and Cortisol Responsiveness following Exposure to Electrical Stimulation versus the Trier Social Stress Tests. PLoS One 7(7): e39375.

[6] Yun Xiang, Huihua Liu, Tiebin Yan, Zhiqiang Zhuang, Dongmei Jin, Yuan Peng. 2014 Functional electrical stimulation-facilitated proliferation and regeneration of neural precursor cells in the brains of rats with cerebral infarction. Neural Regen Res 9(3): 243-251. 